Bangladesh J. Plant Taxon. 17(1): 87-92, 2010 (June)

(C) 2010 Bangladesh Association of Plant Taxonomists

\title{
SOME FRESHWATER PHYTOPLANKTON AS NEW REPORTS AND ONE NEW FORMA FROM BANGLADESH
}

\author{
Md. Almujaddade Alfasane ${ }^{1}$, Md. Shafiqul Islam ${ }^{2}$ and Moniruzzaman KhondKer \\ Department of Botany, University of Dhaka, Dhaka 1000, Bangladesh
}

Keywords: Phytoplankton; Chlamydomonas; Pyramidomonas; Pediastrum; Epicystis; Chroomonas.

\begin{abstract}
Eight newly recorded taxa of freshwater phytoplankton from Bangladesh have been described in the present paper. Of these, 3 species are from Cryptomonadales, 2 species from each of Volvocales and Euglenales and one species from Chromulinales. In addition one new forma of Pediastrum duplex Meyen var. duplex fa. nagdaensis M.A. Alfasane, M.S. Islam \& M. Khondker fa. nov. (Chlorococcales) has also been described in this account.
\end{abstract}

\section{Introduction}

Recently a series of papers have been published where many taxa from different classes have been described and illustrated (Khondker et al., 2007a, b, c; 2008a, b; 2009). During the present study on some recent collections from different parts of the country planktonic algae of different orders have been found to occur which, could not be recorded earlier from the areas selected or any other parts of Bangladesh. So these could be considered as new records for Bangladesh. The recorded species belonged to the genera Chlamydomonas and Pyramidomonas under Volvocales, two taxa of Trachelomonas under Euglenales, Epicystis under Chromulinales and the rest three belonged to Chroomonas, Rhodomonas and Cryptomonas under Cryptomonadales. Pediastrum duplex var. duplex fa. nagdaensis M.A. Alfasane, M.S. Islam \& M. Khondker fa. nov. under Chlorococcales is described as new to science.

\section{Materials and Methods}

Samples for the present paper were collected from 4 different places in Bangladesh. One of them is Buriganga River (near to Sikdar Medical College, Hazaribagh), Dhaka metropolis. In this place, the sampling station (Station 1) is located in the mid-part of the river. The second one is a polluted ditch of village Nagda, Narayanganj. Two other sampling stations are located in two domestic ponds of Pirojpur and Barisal districts (Khondker et al. 2006).

Samples were obtained by sieving $100 \mathrm{~L}$ of sub-surface water through a plankton net having a mesh aperture of $20 \mu \mathrm{m}$. The concentrated plankton sample in the bucket of the plankton net were taken in a screw caped glass vial and fixed with Lugol's solution.

\footnotetext{
${ }^{1}$ Corresponding author. E-mail: mujaddade@yahoo.com

${ }^{2}$ Ansar Academy, Shafipur, Gazipur, Bangladesh.
} 
Organisms were photomicrographed with the help of a Nikon Optiphot, UFX-11A microscope fitted with a Nikon FX-35WA camera, Japan.

\section{Taxonomic enumeration}

A total of eight taxa of freshwater phytoplankton have been identified as new reports for Bangladesh and one taxa has been described as new to science. The illustrated taxonomic descriptions of these taxa are given below.

\section{Division: Chlorophyta; Class: Chlorophyceae; Order: Volvocales;}

Family: Chlamydomonadaceae; Genus: Chlamydomonas

1. Chlamydomonas pomiformis Pascher [Chlamydonephris pomiformis (Pascher) H. and O. Ettl]

(Fig. 1)

(Huber-Pestalozzi, 1961, 159, 28: 139; Dillard, 1989, 16, 1: 18)

Cells spherical to ellipsoidal, posterior end smoothly rounded, anterior truncated, flat, without papilla. Cells $21 \mu \mathrm{m}$ in diameter. Flagella 2, equal, $20 \mu \mathrm{m}$ long.

Buriganga River, Station 1, Collection no. 1-41, 20.05.2005.

\section{Genus: Pyramidomonas}

\section{Pyramidomonas quadricauda Pascher}

(Fig. 4)

(Huber-Pestalozzi, 1961, 13, 3: 17)

Cells strongly metabolic, inverted, ovoid to pear shaped, anterior end rounded, posterior end provided with 4 tentacle like outgrowth. Cells $25 \mu \mathrm{m}$ long, $16 \mu \mathrm{m}$ broad. Flagella 4, $29 \mu \mathrm{m}$ long.

Buriganga River, Station 1, Collection no. 1-41, 20.05.2005.

\section{Order: Chlorococcales; Family: Hydrodictyaceae;}

\section{Genus: Pediastrum}

3. Pediastrum duplex Meyen var. duplex fa. nagdaensis M.A. Alfasane, M.S. Islam \& M. Khondker fa. nov.

(Fig. 2)

Colonia 16 cellularis, $14.73 \mu \mathrm{m}$ diametro, cellulae in colonia circinatim disposita. Cellulae peripheralis $3.81 \mu \mathrm{m}$ longum, $3.56 \mu \mathrm{m}$ latus. Cellulae centralis $2.56 \mu \mathrm{m}$ longum, $3.07 \mu \mathrm{m}$ latus. Distantia inter biapicem 2.56 -3.07 $\mu \mathrm{m}$.

Colony 16-celled, $14.73 \mu \mathrm{m}$ in diameter. Cells in the colony arranged circinally. Peripheral cells $3.81 \mu \mathrm{m}$ long, $3.56 \mu \mathrm{m}$ broad. Central cells $2.56 \mu \mathrm{m}$ long, $3.07 \mu \mathrm{m}$ broad. Distance between two tips 2.56-3.07 $\mu \mathrm{m}$. 

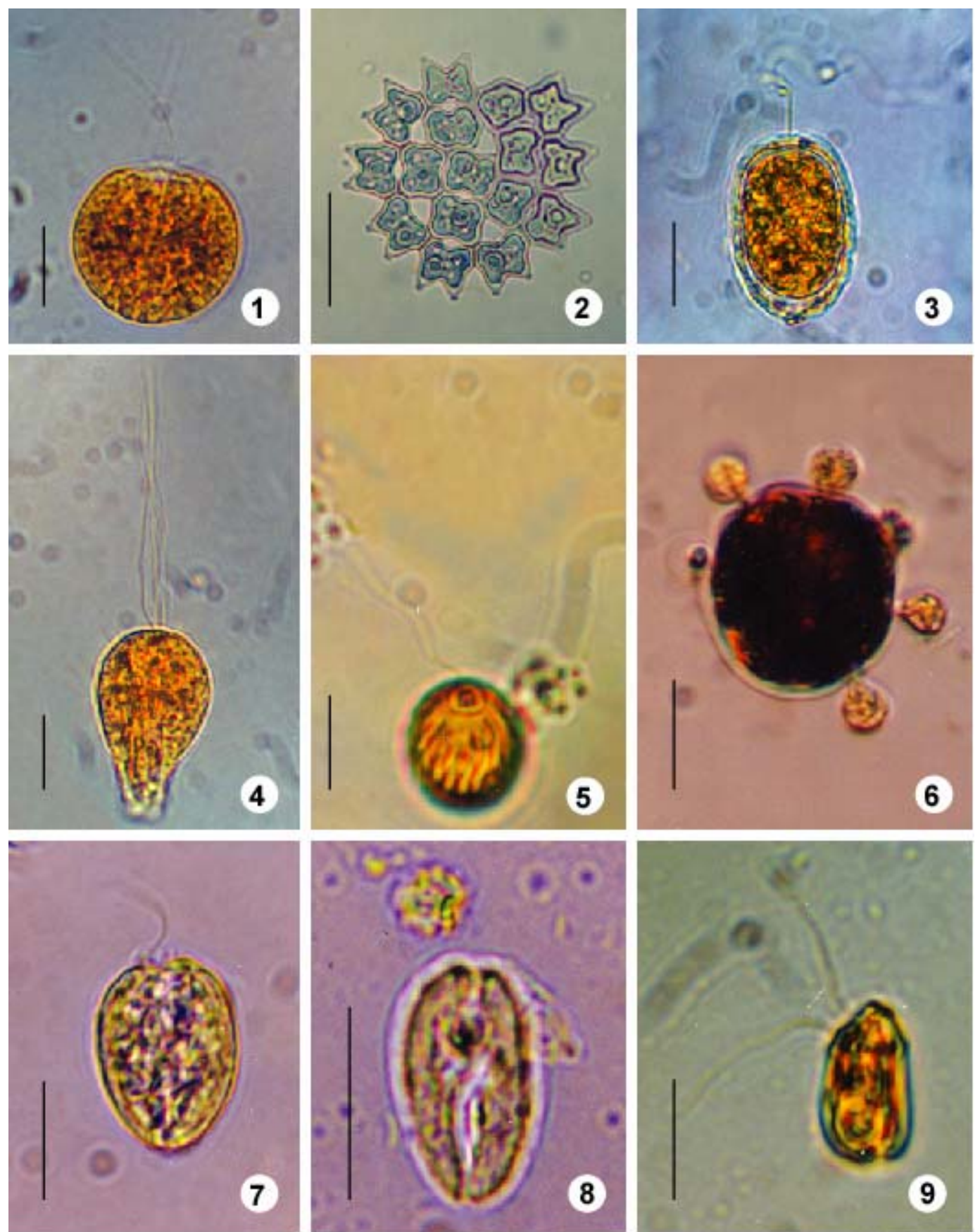

Figs. 1. Chlamydomonas pomiformis Pascher; 2. Pediastrum duplex var. duplex fa. nagdaensis Alfasane et al. fa. nov.; 3. Trachelomonas zorensis Lef.; 4. Pyramidomonas quadricauda Pascher; 5. Trachelomonas stockesiana var. conradi (Defl.) H.-P.; 6. Epicystis peridinearum Pascher (epiphytic on Peridinium cyst); 7. Chroomonas pochmani Huber-Pestalozzi; 8. Rhodomonas ovalis Nygaard; 9. Cryptomonas alpina Chodat. Bars $=10 \mu \mathrm{m}$. 
Notes: This new forma differs from the typical by being smaller in colony diameter and length-breadth ratio of central and peripheral cells. In the type, peripheral cell length 1.42 times more than breadth, in the central cells it is nearly double (Huber-Pestalozzi, 1983). In this new forma, length and breadth ratio of both peripheral and central cells are 1.07 and 0.83 , respectively.

Holotype: Sample no. 7(2), 17 July 2003, Nagda, Narayanganj district, collected by Mohammed Almujaddade Alfasane, Herbarium of Phycology, Hydrobiology and Limnology Laboratory, Department of Botany, University of Dhaka, Dhaka, Bangladesh.

Type locality: The sample was collected from a polluted ditch located in the village Nagda, Narayanganj, Bangladesh, $Z_{\max }=1.7 \mathrm{~m}, A=1600 \mathrm{~m}^{2}$. The ditch receives effluents from the nearby industries.

Ecology: Pelagic plankton, water $\mathrm{pH}$ was 7.67.

\section{Division: Euglenophyta; Order: Euglenales; Family: Euglenaceae; Genus: Trachelomonas}

4. Trachelomonas stockesiana var. conradi (Defl.) Huber-Pestalozzi [T. rugulosa bei Conrad, T. conradi Skv., T. rugulosa var. conradi Defl.]

(Fig. 5)

(Huber-Pestalozzi, 1955, 265, 58: 410A)

Lorica elliptical, flagellum aperture with a thick ring. Membrane strongly rugose. Cells $14 \mu \mathrm{m}$ long, $11.4 \mu \mathrm{m}$ broad. Flagellum $17.8 \mu \mathrm{m}$ long.

Bakerganj, Station No. 2, Collection no. NHEB0410042, 15.06.2004.

\section{Trachelomonas zorensis Lef.}

(Fig. 3)

(Huber-Pestalozzi, 1955, 320, 69: 627b)

Lorica ellipsoidal, posterior end broadly rounded. Flagellum aperture without a collar but with ring like thickening. Cell membrane thick with fine scrobiculations, yellowish brown. Lorica $23 \mu \mathrm{m}$ long, $14 \mu \mathrm{m}$ broad. Flagellum about twice the length of the body.

Bakerganj, Station No. 4, Collection no. NHEB0410054, 12.07.2004.

\section{Division: Chrysophyta; Order: Chromulinales;}

Family: Chrysosphaeraceae; Genus: Epicystis

6. Epicystis peridinearum Pascher

(Fig. 6)

(Kristiansen and Preisig, 2001, 120-121, 89).

Unicellular, solitary. Cells spherical, thin walled, epiphytic on Peridinium cysts, 5 $\mu \mathrm{m}$ in diameter.

Bakerganj, Station No. 4, Collection no. NHEB0510124, 27.01.2005. 


\section{Division: Cryptophyta; Order: Cryptomonadales; Family: Cryptomonadaceae; Genus: Chroomonas}

7. Chroomonas pochmani Huber-Pestalozzi

(Fig. 7)

(Huber-Pestalozzi, 1968, 31, 3: 16a,b)

Unicellular, solitary, free swimming. Cells broadly oval, anterior end broadly rounded, posterior end slightly attenuated. Cells $17 \mu \mathrm{m}$ long, median diameter $12 \mu \mathrm{m}$. Flagella 2, short, almost equal to body length.

Narayanganj, Station No. 5, Collection no. 5, 17.07.2003.

\section{Genus: Cryptomonas}

8. Cryptomonas alpina Chodat

(Fig. 9)

(Huber-Pestalozzi, 1968, 66, 8: 52)

Unicellular, solitary, slipper shaped. Cells elongated, posterior end flatly rounded, anterior one slightly depressed in angle. Flagella 2, $12 \mu \mathrm{m}$ long. Cells $13 \mu \mathrm{m}$ long, $8 \mu \mathrm{m}$ broad.

Mathbaria, Station No. 3, Collection no. NHEM0460053, 21.06.2004.

\section{Genus: Rhodomonas}

9. Rhodomonas ovalis Nygaard

(Fig. 8)

(Huber-Pestalozzi, 1968, 25: 11A, c)

Unicellular, solitary, free swimming. Cells ovoid to elongated oval-cylindric, two times longer than broad. Pyrenoid single, present in anterior part. Cells $15 \mu \mathrm{m}$ long, 8 $\mu \mathrm{m}$ broad.

Narayanganj, Station No. 5, Collection no. 5, 17.07.2003.

\section{Acknowledgements}

The research as an integral part of the major multidisciplinary project entitled 'Epidemiology and Ecology of Vibrio cholerae in Bangladesh' was financed by the National Institute of Health (NIH) research grant No. 1RO1A13912901 under the collaborative agreement between the International Centre for Diarrhoeal Disease Research, Bangladesh (ICDDR,B) and Johns Hopkins Bloomberg School of Public Health. The authors gratefully acknowledge the NIH Ecological Surveillance Team at ICDDR,B for kindly supporting this research (i.e. collections of Bakerganj and Mathbaria). Latin diagnosis of the new forma done by Prof. Syed Hadiuzzaman, Department of Botany, University of Dhaka is also gratefully acknowledged. 


\section{References}

Dillard., G.E. 1989. Freshwater Algae of Southern United States. Part 1. Chlorophyceae: Volvocales, Tetrasporales and Chlorococcales. Bibl. Phycol. Vol. 81. J. Cramer.Berlin, pp. $202+$ pls. 37.

Huber-Pestalozzi, G. 1955. Das Phytoplankton des Süßswassers: Systematik und Biologie. Euglenophyceen E. Schweizerb. Verlagsb. (Nägele u. Obermiller), Stuttgart, Germany. pp. $606+$ pls. 114.

Huber-Pestalozzi, G. 1961. Das Phytoplankton des Süßswassers. Systematik und Biologie. 5. Teil: Chlorophyceae (Grünalgen), Ordnung: Volvocales. E. Schweizerb. Verlagsb. (Nägele u. Obermiller), Stuttgart, Germany. pp. 744 + pls. 157.

Huber-Pestalozzi, G. 1968. Das Phytoplankton des Süsswassers. Systematik und Biologie. 3. Teil: Cryptophyceae, Chloromonadophyceae, Dinophyceae. E. Schweizerbart'sche Verlagsbuchhandlung (Nägele u. Obermiller), Stuttgart, Germany. 322 pp.

Huber-Pestalozzi, G. 1983. Das Phytoplankton des Süsswassers. Systematik und Biologie. 7. Teil, 1. Hälfte, Chlorophyceae (Grünalgen) Ordnung Chlorococcales. E. Schweizerbart'sche Verlagsbuchhandlung (Nägele u. Obermiller), Stuttgart, Germany. 1400 pp.

Khondker, M., Bhuiyan, R.A., Yeasmin, J., Alam, M., Sack, R.B., Huq, A. and Colwell, R.R. 2006. New records of phytoplankton for Bangladesh. 1. Cyanophyceae. Bangladesh J. Bot. 35(2): 173-179.

Khondker, M., Bhuiyan, R.A., Yeasmin, J., Alam, M., Sack, R.B., Huq, A. and Colwell, R.R. 2007a. New records of phytoplankton for Bangladesh. 2. Cryptophyceae and Synurophyceae. Bangladesh J. Bot. 36(1): 53-59.

Khondker, M., Bhuiyan, R.A., Yeasmin, J., Alam, M., Sack, R.B., Huq, A. and Colwell, R.R. 2007b. New records of phytoplankton for Bangladesh. 3. Order: Volvocales. Bangladesh J. Plant Taxon. 14(1): 1-12.

Khondker, M., Bhuiyan, R.A., Yeasmin, J., Alam, M., Sack, R.B., Huq, A. and Colwell, R.R. 2007c. New records of phytoplankton for Bangladesh. 4. Order: Chlorococcales. Bangladesh J. Plant Taxon. 14(2): 83-91.

Khondker, M., Bhuiyan, R.A., Yeasmin, J., Alam, M., Sack, R.B., Huq, A. and Colwell, R.R. 2008a. New records of phytoplankton for Bangladesh. 5. Euglena, Euglenocapsa. Bangladesh J. Plant Taxon. 15(1): 39-46.

Khondker, M., Bhuiyan, R.A., Yeasmin, J., Alam, M., Sack, R.B., Huq, A. and Colwell, R.R. 2008b. New records of phytoplankton for Bangladesh. 8. Trachelomonas Ehr. (Euglenophyceae). Bangladesh J. Bot. 37(2): 133-139.

Khondker, M., Bhuiyan, R.A., Yeasmin, J., Alam, M., Sack, R.B., Huq, A. and Colwell, R.R. 2009. New records of phytoplankton for Bangladesh. 9. some rare and a new species. Bangladesh J. Plant Taxon. 16(1): $1-8$.

Kristiansen, J. and Preisig, H.R. (eds). 2001. Encyclopedia of Chrysophyte Genera. Bibl. Phycologica 110: $120-121$ 